\title{
Integrated Modeling for the Design of Deformable Mirrors Using a Parametric Module Method
}

\author{
Junqing Zhu ${ }^{1,2 *}$, Wei Sha ${ }^{1}$, Changzheng Chen ${ }^{1}$ Xingxiang Zhang $^{1}$, and Jianyue Ren ${ }^{1}$ \\ ${ }^{1}$ Changchun Institute of Optics, Fine Mechanics and Physics, Chinese Academy of Sciences, \\ Changchun 130033, China \\ ${ }^{2}$ University of Chinese Academy of Sciences, Beijing 100049, China
}

(Received June 26, 2015 : revised August 13, 2015 : accepted August 17, 2015)

\begin{abstract}
Active optics is a key technology for future large-aperture space telescopes. In the design of deformable mirrors for space applications, the design parameter trade-off between the number of regularly configured actuators and the correction capability is essential but rarely analyzed, due to the lack of design legacy. This paper presents a parametric module method for integrated modeling of deformable mirrors with regularly configured actuators. A full design parameter space is explored to evaluate the correction capability and the mass of deformable mirrors, using an autoconstructed finite-element parametric modeling method that utilizes manual finite-element meshing for complex structures. These results are used to provide design guidelines for deformable mirrors. The integrated modeling method presented here can be used for future applied optics projects.
\end{abstract}

Keywords : Active optics, Space telescope, Deformable mirrors, Integrated modeling, Wavefront compensation OCIS codes : (220.1000) Aberration compensation; (220.1080) Active or adaptive optics; (230.4040) Mirrors; (220.4880) Optomechanics; (350.6090) Space optics

\section{INTRODUCTION}

With the increasing demand for earth observations and astronomy surveys, space telescopes have been driven to evolve towards designs with large apertures, wide fields of view, and agile observation. However, the optomechanical design of large-aperture space telescopes is still one of the most challenging tasks in space projects.

The difference in gravity between fabrication on Earth and operation in space leads to a constant bias in optical structure. Image quality degradation occurs due to the change in optical structure and the figure error of optical surfaces, which are caused by gravitational effects, thermoelastic distortion, and structural vibration. A rational optomechanical design of a space telescope is required to maintain stability of the optical structure under environmental disturbance. Meanwhile, both weight and size restrictions should be met for space applications; therefore, a lightweight design is essential for large-aperture space telescopes [1].

For small-aperture optics, high system stiffness can be obtained by using a lightweight structure and a material with high Young's modulus [2]. Image quality degradation due to the changes in environment will be acceptably controlled by a proper design and tolerance budget.

Unlike small-aperture optics, system weight and size will count against the lightweight design if large-aperture optics also pursue a high-system-stiffness design to guarantee optical structure stability. The constraints of launch weight and size are violated, which makes the system unachievable. On the other hand, the fact that large-aperture optics are more sensitive to gravitational effects and thermoelastic and structural vibrations makes it even harder to design for space applications [3-5].

Therefore, to achieve a very lightweight design with excellent performance, large-aperture optics must use active methods, namely active optics, to correct optomechanical constant bias, misalignment, and system wavefront error caused by environmental issues. Active-optics technology has only recently been applied to space telescopes. For example, the James Webb Space Telescope (JWST), which is planned to be launched in 2018, will be implemented with multiple active-optics sets. With an active primary mirror whose diameter is 6.5 $\mathrm{m}(\mathrm{F} / 1.2)$, the JWST reaches the diffraction limit at a wave-

\footnotetext{
*Corresponding author: zhujunqing2014@163.com

Color versions of one or more of the figures in this paper are available online.
} 
length of $2 \mu \mathrm{m}$ [6].

Space active optics mainly has the following two solutions in application.

One approach uses an active primary mirror, such as in the Hubble Space Telescope (HST) and the JWST mentioned above. The HST's primary mirror has 24 actuators to compensate for lower-order aberrations, while the JWST's primary mirror consists of 18 mirror segments, each with 7 degrees of freedom to compensate for rigid body movements and curvature change. The current state-of-the-art active primary mirror is the actuated hybrid mirror (AHM) developed by the Jet Propulsion Laboratory (JPL) [7], with a fast fabrication process, low areal density, and wavefront-correction capability making it promising for an extreme large-aperture space telescope [8].

The other approach uses exit-pupil deformable mirrors (DMs). Given that the aperture stop is on the primary mirror [9], which is more sensitive than other optical elements to environmental loads due to its size, a real exit pupil is required where a small, deformable mirror is placed to be conjugate to the primary mirror and to compensate for its figure error and the system wavefront error. This kind of DM is studied in this paper.

Unlike adaptive optics applied in large ground-based observatories, space active optics is dedicated to compensating for lower-order and larger-amplitude optical aberrations, and works at much lower frequency [10]. Existing DMs cannot be directly used in space applications because of operation specifications and critical restrictions for reliability and weight, size, power consumption, etc. For space applications, multidisciplinary constraints should be addressed, and a lot of effort is needed to make a technological breakthrough for DMs in future large-aperture space telescopes.

In general, the more actuators a deformable mirror has, the more degrees of freedom, and the better correction capability it can achieve. Due to the absence of design heritage, the design parameter trade-off that is essential to the design of DMs is still unexplored, and the optimal design of a DM is unknown, up to now.

S. K. Ravensbergen [11] developed a semianalytical model (ignoring edge effects) to describe thin facesheets on discrete actuators, using the plate theory of elasticity. However, this model is limited in providing a starting point, as its data is not suitable for further analysis. An analytic approach for DM design is difficult due to the lack of proper theory.

A parametric modeling tool [12] has been developed by the Space Systems Laboratory (SSL) at MIT to assist in the preliminary design of next-generation, lightweight space telescopes. The automatic-construction method in that tool for modeling an active-segment mirror is a valuable approach for parametric modeling [13], although the method is only available for simple structures and when low-fidelity finite elements are used.

NASA's new mirror-modeling software [14] can automatically generate both mirror and suspension-system elements in several minutes, which makes the integration of these models into large telescope or satellite models easy. However, this software can only deal with a predetermined substrate pattern, and a relatively simple structure model.

Due to the lack of fully developed automeshing technology [15], high-fidelity finite-element models (FEM) are still built by manual meshing, which is time-consuming and impractical for large-volume design of experiments (DOE). The existing autoconstruction method is quite limited to simple and regular structures [16], using low-fidelity elements for many parts (e.g. triangular elements or tetrahedral elements).

To provide design guidelines for DMs, a new parametric module method for integrated modeling of a DM with regularly configured actuators is presented, by using an autoconstruct parametric finite-element modeling method that takes advantage of manual finite-element meshing for complex structures. The method is practical for other applications as well.

The paper is organized as follows: Section 2 presents the large-aperture space telescope and the parametric module method. Section 3 describes the details of application of the parametric module method to the DM parametric modeling. Section 4 describes the integrated modeling process of DM figure compensation and the metric of correction capability. Section 5 presents the design trade-off carried out on a DM $320 \mathrm{~mm}$ in diameter, and Section 6 concludes the paper.

\section{LARGE-APERTURE SPACE TELESCOPE AND PARAMETRIC MODULE METHOD}

\subsection{Large-Aperture Space Telescope Description}

The large-aperture space telescope employs a 5-mirror telescope design with three off-axis mirror anastigmatic (TMA) optics and a focal length of $20 \mathrm{~m}, \mathrm{~F} / 10$, as shown in Fig. 1. The primary mirror is a monolithic light-weighted mirror of $\Phi=2.5 \mathrm{~m}$, conjugated with an exit-pupil mirror $320 \mathrm{~mm}$ in diameter. Although the primary mirror is made of silicon carbide $(\mathrm{SiC})$, which has a very high stiffness-todensity ratio, the primary mirror is deformed dramatically under gravity due to its size. The wavefront error (WFE) of the primary mirror for $1 \mathrm{~g}$ of gravity is pictured in Fig. 2(a) with $802 \mathrm{~nm}$ pv, $162 \mathrm{~nm}$ rms. The WFE is decomposed

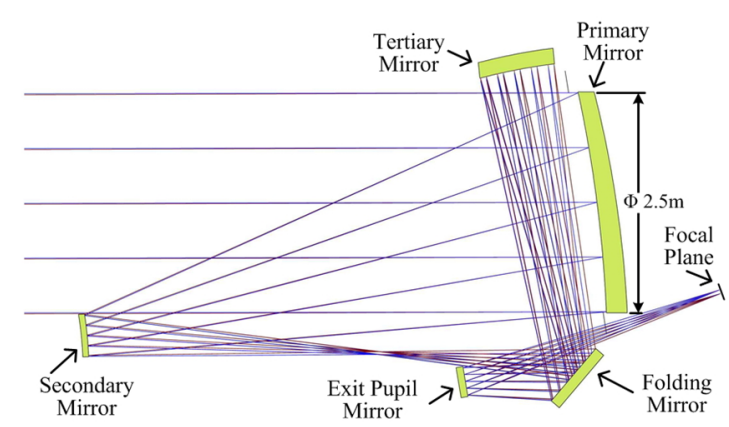

FIG. 1. Optical configuration of a large-aperture space telescope. 
into Zernike polynomials as described in Fig. 2(b), which shows that trefoil, spherical, and hexagonal modes of Zernike polynomials are mainly involved, due to the structure of the mirror's mount. The significant Zernike terms are labeled and listed in Table 1. The WFEs for selected Zernike modes

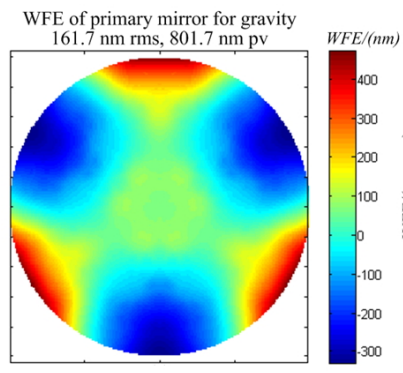

(a)

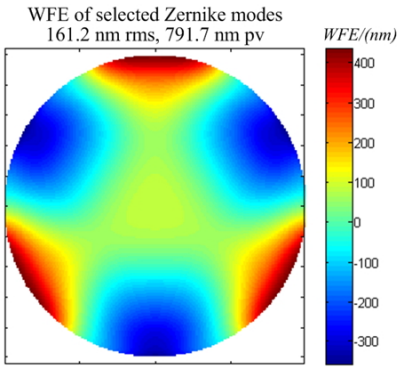

(c)

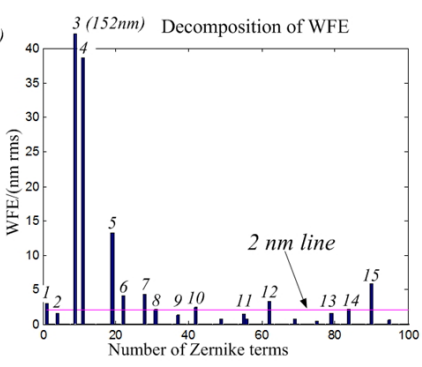

(b)

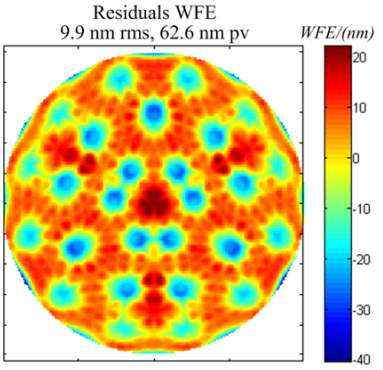

(d)
Residuals WFE

FIG. 2. WFE of the primary mirror due to gravity: (a) WFE of the primary mirror due to gravity. (b) Decomposition of the WFE; labeled terms of Zernike polynomials are listed in Table 1. (c) WFEs of selected Zernike terms listed in Table 1. (d) Corresponding residual WFEs of selected Zernike terms.

TABLE 1. Main components of the WFE of the primary mirror for gravity

\begin{tabular}{c|c|c|c|c}
\hline \hline Number & $\mathrm{n}$ & $\mathrm{m}$ & Mode & WFE (nm rms) \\
\hline 1 & 0 & 0 & Bias & 3.1 \\
\hline 2 & 2 & 0 & Power & 1.6 \\
\hline 3 & 3 & 3 & Trefoil5 & 152.0 \\
\hline 4 & 4 & 0 & Spherical3 & 38.7 \\
\hline 5 & 5 & -3 & Trefoil7 & 13.2 \\
\hline 6 & 6 & 0 & Spherical5 & 4.2 \\
\hline 7 & 6 & 6 & Hexafoil11 & 4.3 \\
\hline 8 & 7 & -3 & Trefoil9 & 2.3 \\
\hline 9 & 8 & 0 & Spherical7 & 1.3 \\
\hline 10 & 8 & 6 & Hexafoil13 & 2.4 \\
\hline 11 & 9 & -9 & Enneafoil & 1.5 \\
\hline 12 & 10 & -6 & Hexafoil15 & 3.4 \\
\hline 13 & 12 & 0 & Spherical11 & 1.6 \\
\hline 14 & 12 & 6 & Hexafoil17 & 2.2 \\
\hline 15 & 12 & 12 & Dodecafoil & 5.9 \\
\hline & & & &
\end{tabular}

are presented in Fig. 2(c), with the residual WFE shown in Fig. 2(d). The residual WFE comprises much higher-order terms of Zernike polynomials and its value is within $\lambda / 50$ $\mathrm{rms}, \lambda 10 \mathrm{pv}(\lambda=632.8 \mathrm{~nm})$. To meet the required tolerance for the primary mirror, the exit-pupil mirror is a DM with an effective aperture of $\Phi=320 \mathrm{~mm}$, and the residual WFE after correction of the deformation of the primary mirror due to gravity should be less than $\lambda / 50 \mathrm{rms}$.

\subsection{DM Design and Parametric Module Method}

The structure of a continuous facesheet DM with discrete surface-normal actuators is described in Fig. 3. The desired figure on the mirror is generated by commanding actuators to work in a specified manner. Inner stress and the actuator print-through effect are relieved by using the Pad and Flexure. Generally, because of fabrication concerns, actuators of a $\mathrm{DM}$ are configured in a regular pattern; see Fig. 4. Moreover, note the dashed lines across the mirror; it is clear that the mirror can be divided into similar parts.

Figure 5 shows the approach to Parametric Module Modeling. Based on the fact that a DM with complex structure can be divided into repeatable parts, similar parts are defined by a module that is modeled by manual meshing and parametrized into a Parametric Module by using the object-oriented programming method. DM modules with similar Properties and Methods are defined by a Class, which is easily organized and operated.

Properties of the DM Class designed for the DM modules consist of the Parameter that presents the dimensions of

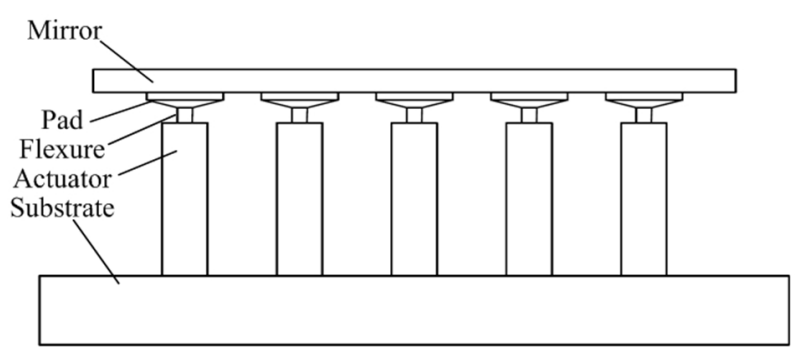

FIG. 3. Structure of a continuous DM with discrete surfacenormal actuators.

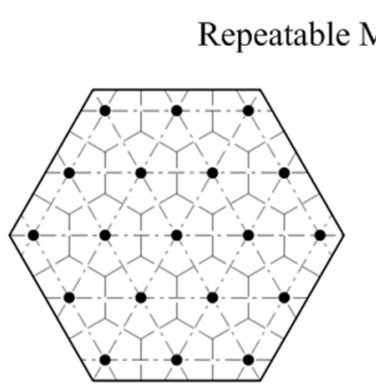

(a)

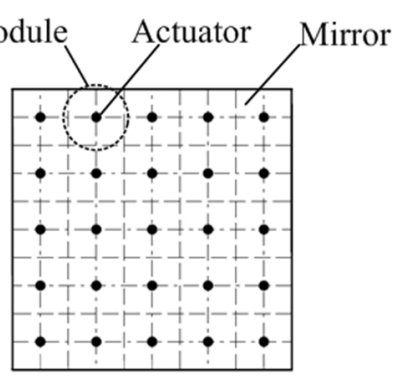

(b)
FIG. 4. Two different patterns of actuators: (a) triangularly configured DM, (b) squarely configured DM. 


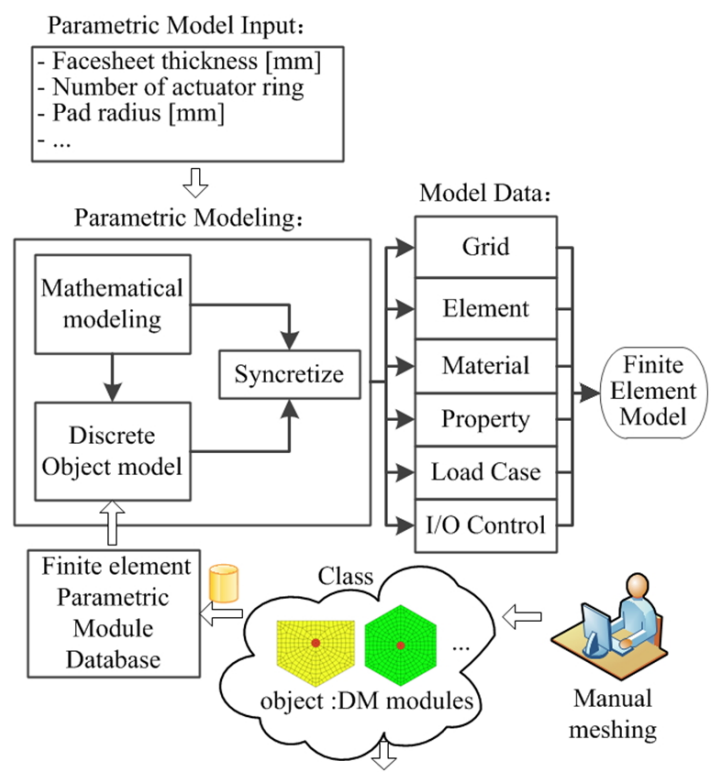

FIG. 5. Finite-element parametric module modeling process.

the module, the FEM information including FEM data for the module (e.g. information about Grid, Element, Components, SPC, etc.), and the Accessory that stores the Local coordinates, the Interface of FEM, and the Node/Element-Sets used in operations to declare each part of the module.

Methods, i.e. the operations that can be performed on the DM modules, are specified as functions, including the rigid transformation of FEM, the mirror operation of FEM, the parameter update of FEM, etc.

Managing the complexity of large modeling applications is improved by applying an object-oriented approach. By using parametric module modeling, discrete parts of the DM are generated from the DM modules defined by the DM Class and operated to possess a calculated position, orientation, and dimensions. The FEM of the DM is constructed by syncretizing the FEM of all parts. According to the solver deck used in finite-element analysis, the FEM of the DM is written into an FEM input file in a specified format, and finite-element analysis is then conducted.

The parametric method is useful for integrated modeling of complex structured models by combining manual finiteelement meshing with the autoconstructed meshing method. The parametric and automated nature of this method allows large-volume design iteration.

\section{DM MODELING APPROACH}

The parameters of the DM used in this paper are illustrated in Fig. 6. They consist of the effective aperture size $L$, the facesheet thickness $T$, the pad diameter $\phi$, and the number of actuator rings $N$. Baseline mirror parameters are listed in Table 2.

The DM Class is designed according to the character of

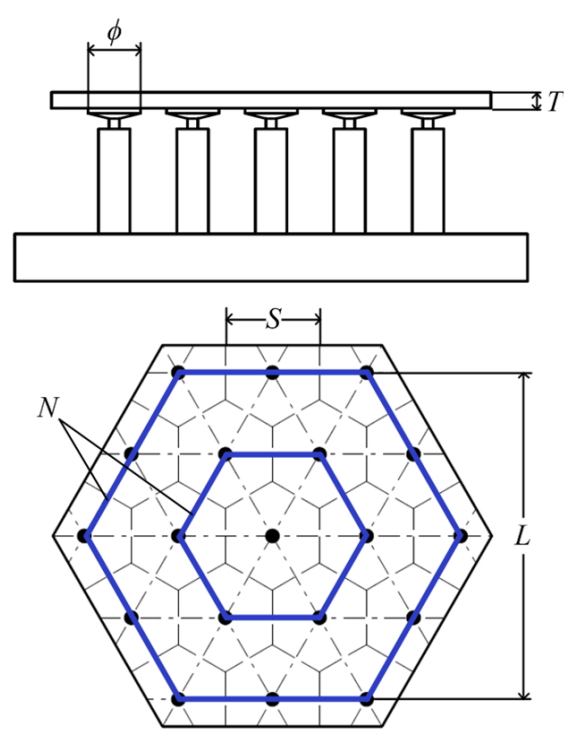

FIG. 6. Parameters of the DM.

TABLE 2. Baseline for DM parameters

\begin{tabular}{c|c|c|c}
\hline \hline Parameter & Units & $\begin{array}{c}\text { Baseline } \\
\text { value }\end{array}$ & Range \\
\hline Effective aperture size $(L)$ & $\mathrm{mm}$ & 320 & fixed \\
\hline Facesheet thickness $(T)$ & $\mathrm{mm}$ & 2 & $0.4-5$ \\
\hline Pad diameter $(\phi)$ & $\mathrm{mm}$ & 4 & $4-12$ \\
\hline Number of actuator rings $(N)$ & - & 4 & $2-16$ \\
\hline
\end{tabular}

the DM module. Details of the DM Class and the whole model-building procedure are explained as follows.

\subsection{Properties of DM Class}

Figure 7 shows the FEM of several DM modules. It is clear that these modules have common structural features and parameters; thus, the properties of the DM Class are defined.

The independent dimensions of the DM module are stored in the Property Parameter, which includes the Character size $S$, the Mirror substrate thickness $T$, and the Pad diameter $\phi$. Table 3 shows the baseline for the DM module parameters. The parameters $S$ and $\phi$ are inherited from the whole DM. The parameter $S$ is determined by $L, N$, and the actuator configuration of the DM. For example, the character size $S$ for a triangularly configured DM is calculated as:

$$
S=\sqrt{3} L /(2 N+3)
$$

The Property FEM Info reserves information describing the FEM (e.g. information about Grid, Element, Components, SPC, etc.). The manually meshed FEM possesses good element quality as well as high fidelity, by using hexagonal elements; see Fig. 7. Note that the boundaries of FEM for one pair 


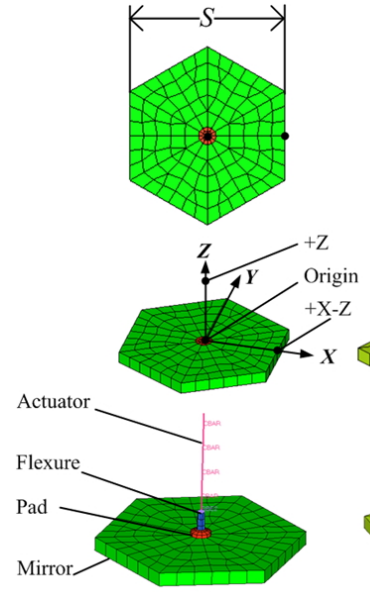

(a)
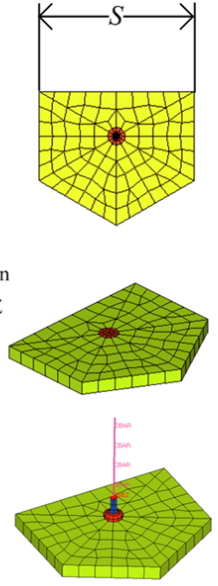

(b)
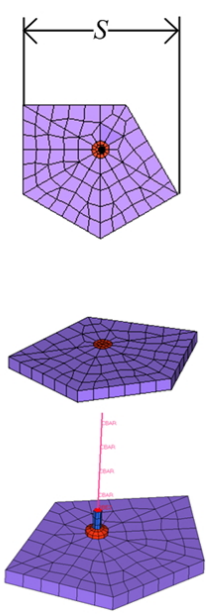

(c)
FIG. 7. Finite-element model of several DM modules.

TABLE 3. Baseline for DM module parameters

\begin{tabular}{c|c}
\hline \hline Parameter & Baseline value \\
\hline Facesheet size $(S)$ & $25 \mathrm{~mm}$ \\
\hline Facesheet thickness $(T)$ & $2 \mathrm{~mm}$ \\
\hline Pad diameter $(\phi)$ & $4 \mathrm{~mm}$ \\
\hline
\end{tabular}

of DM modules are consistent with each other, to ensure that the edge nodes of different parts of the FEM stay within tolerance before equivalent nodes are merged.

The Property Accessory contains the Local coordinate of the DM, the Interface of the FEM, and the Node/Element Sets to be used in operations by declaring parts of the DM module. The local coordinate is determined by three points: the origin point of the local coordinate, a point on the $+Z$ axis, and a point on the $+X-Z$ plane. The local coordinate does not need to be the same as the global coordinate in which the FEM is defined. It is useful for ensuring that all parts of the DM model are consistent. Node/Element Sets are used for grouping parts of the FEM as components to make FEM operations possible.

\subsection{Methods of DM Class}

Driven by the application of the DM module, several Methods are designed to update the FEM Info of the DM module according to the associated parameters. The Methods used in this paper include:

- The rigid transformation of FEM

- The mirror operation of FEM

- The parameter update of FEM

The Methods change the nodal coordinates of the DM module by transforming or scaling the associated Node/Element Sets that are stored in the property of the DM module. Note that the method of scaling the FEM is limited according

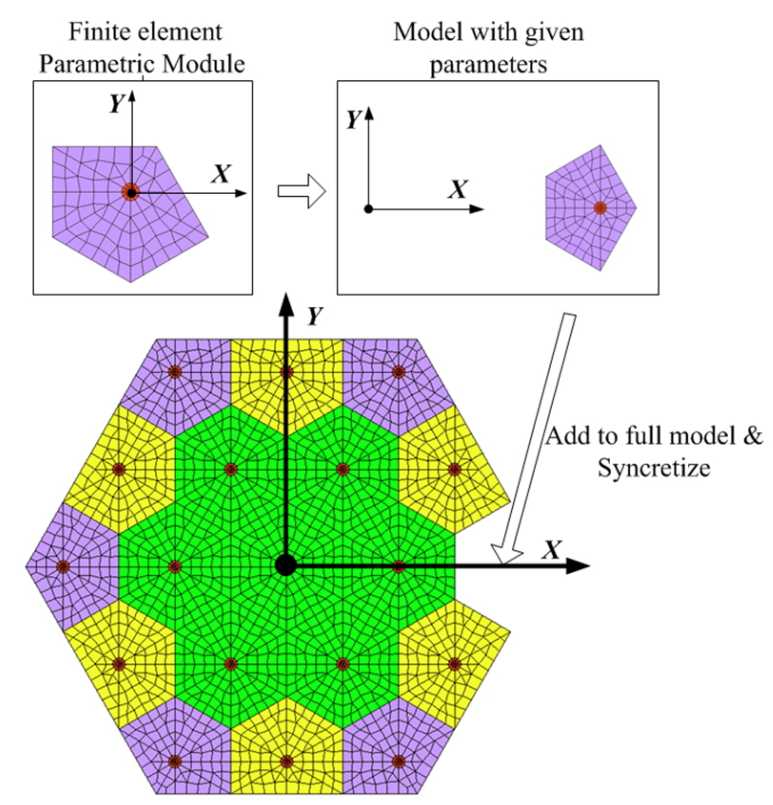

FIG. 8. Autoconstruction process of a DM based on modules.

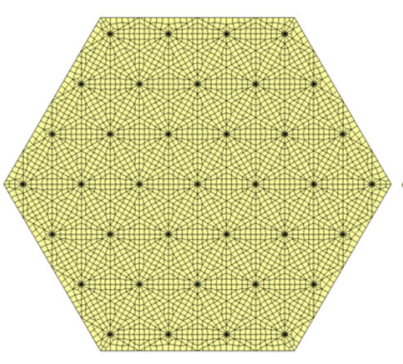

(a)

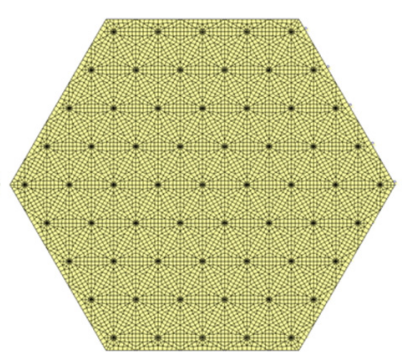

(b)
FIG. 9. DM with (a) $N=3$ and (b) $N=4$.

to the element uniformity of the DM FEM. To obtain high fidelity in analysis, a set of DM module pairs with different mesh densities are needed in application. Moreover, the Methods defined in the DM Class can be extended for future applications.

\subsection{DM Assembly}

Once the DM modules have been built according to the definition of the DM Class, the DM model can be constructed by assembling all parts of the DM that are computed from corresponding DM modules. See Fig. 8. For example, one part of the DM model is updated from the DM module by modifying its dimensions, location and orientation predefined for the whole model. Then, through synthesis, this part is added to the whole DM model. According to the solver deck being used, the whole DM model and associated boundary conditions are written into an FEM input file in the specified format. Given the desired parameters, it takes mere minutes to construct the DM model. Figure 9 shows two DM models made up of different numbers $N$ of actuator rings. 


\section{DM CORRECTION CAPABILITY ANALYSIS}

Shape control based on influence function has been chosen as the standard. It is divided into two types: the Zernike influence function and the Nodal influence function. It is convenient to acquire the Nodal influence function by extracting the surface nodes' displacements from the results of finite-element analysis in which each actuator is given a unit command. The Zernike influence function has a spatial bandwidth limitation, and any portion of the shape disturbance that lies above the bandwidth of a set of Zernike polynomials is missed, so the residual error of Zernike fitting for the shape disturbance may be large and unacceptable[17]. The Nodal influence function is preferred in this paper, since computational cost is not a concern in this research.

The integrated modeling approach to DM correction capability analysis is illustrated in Fig. 10. Based on the parametric module method modeling described above, after a "unite" input is given to each actuator, the influence functions are obtained, and then the commands of object figures and the correction capability criterion are calculated.

Consider $m$ surface nodes distributed on the surface of the DM FEM with $n$ actuators. Then the Nodal influence function $\boldsymbol{\psi}_{i} \in \mathfrak{R}^{m}$ for the $i$ th actuator is obtained from the nodal deflections of the DM surface, by inputting the "unite" command to the $i$ th actuator while all other actuators are given zero input. The influence function matrix is given by assembling all Nodal influence functions $\boldsymbol{\psi}_{i}$ :

$$
\boldsymbol{\Psi}=\left[\begin{array}{llll}
\boldsymbol{\Psi}_{1} & \boldsymbol{\Psi}_{2} & \cdots & \boldsymbol{\Psi}_{n}
\end{array}\right] \in \mathfrak{R}^{m \times n}
$$

The WFE of the primary mirror due to gravity is chosen as the object figure to estimate the DM cDM correction capability. The residual WFE should be controlled to within $\lambda 50$ rms. Object figure $\mathbf{b} \in \mathfrak{R}^{m}$ is calculated by interpolating primary mirror figure deformation due to gravity at associated nodal points of the DM surface that are normalized to the unit circle.

It is assumed that the DM is a linear system when deflections are much smaller than the size of the DM, and the surface figure of the DM under actuation can be obtained by superimposing surface figures under the motions of associated actuators. Meanwhile, the surface nodes of the DM FEM are distributed nonuniformly, so a weighting matrix $\mathbf{W}$ is used to address the area weight for each node. Hence, the

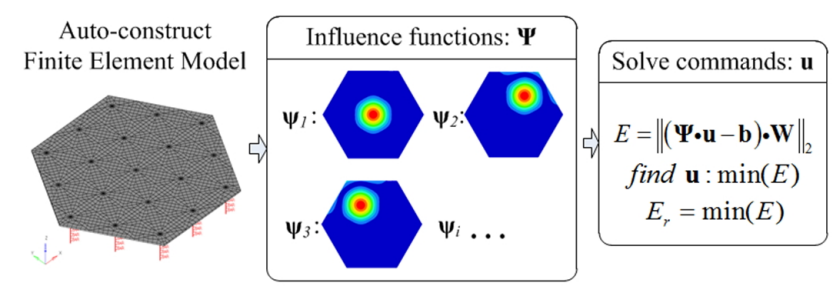

FIG. 10. Calculation process of DM correction capability. control vector $\mathbf{u}$ containing commands for associated actuators, can be computed via the influence matrix by minimizing the RMS of residual deflection of a figure, $E_{r}$, which is the metric for DM correction capability.

$$
\begin{aligned}
& E=\|(\boldsymbol{\Psi} \cdot \mathbf{u}-\mathbf{b}) \cdot \mathbf{W}\|_{2} \\
& \text { find } \mathbf{u}: \min (E), \quad E_{r}=\min (E)
\end{aligned}
$$

\section{DM DESIGN TRADE SPACE ANALYSIS}

There are 4 design parameters $(\mathrm{L}, L, T, \phi, N)$ for a specified configuration of a DM. The parameter $L$ is fixed for a specific application (here $320 \mathrm{~mm}$ ). In addition to the correction capability mentioned in the previous section, the mass of a DM is another metric of design, as launch mass is extremely limited for space applications. The materials used in this paper for each associated component are listed in Table 4. The main mass contributors are the mirror and the PZT actuator assemblies (PZT actuator plus pad and flexure). The mass of the actuator assemblies can be assessed by multiplying the number of actuators by the mass of a single actuator assembly, which is $10 \mathrm{~g}$ in general.

A full trade space of designs is enabled by integrated modeling. There are three design parameters and two independent metrics (correction capability and mass). Via full-level design of experiments (DOE), all possible design cases can be evaluated.

\subsection{Design Trade Space Analysis}

The triangularly configured DM is analyzed first. By fixing $L=320 \mathrm{~mm}$ and then varying $N$ from 2 to $16, \phi$ from $4 \mathrm{~mm}$ to $6 \mathrm{~mm}$, and $T$ from $0.4 \mathrm{~mm}$ to $5 \mathrm{~mm}$, the trade space is analyzed via full-level design of experiments (DOE). The results are illustrated in Fig. 11. Each point is a design, differentiated by the parameter $N$. The ideal design of a DM is one with both the best correction capability and the smallest mass, while the correction capability metric $E_{r}$ decreases as the number of actuators (indicated by $N$ ) increases, which also adds to the mass of the DM. The best design is affected by the trade-off between $E_{r}$ and the mass. The designs on the low frontier of the trade space make up the Pareto Optimal Designs listed in Table 5. The Pareto Front is plotted as a green line.

TABLE 4. Materials properties used in DM design

\begin{tabular}{c|c|c|c}
\hline \hline & Young's modulus & Density & Components \\
\hline Units & Gpa & $\mathrm{g} / \mathrm{cm}^{3}$ & \\
\hline ULE & 91 & 2.53 & Mirror, Pad \\
\hline Titanium & 114 & 4.40 & Flexure \\
\hline PZT & 75 & 7.80 & Actuator \\
\hline
\end{tabular}




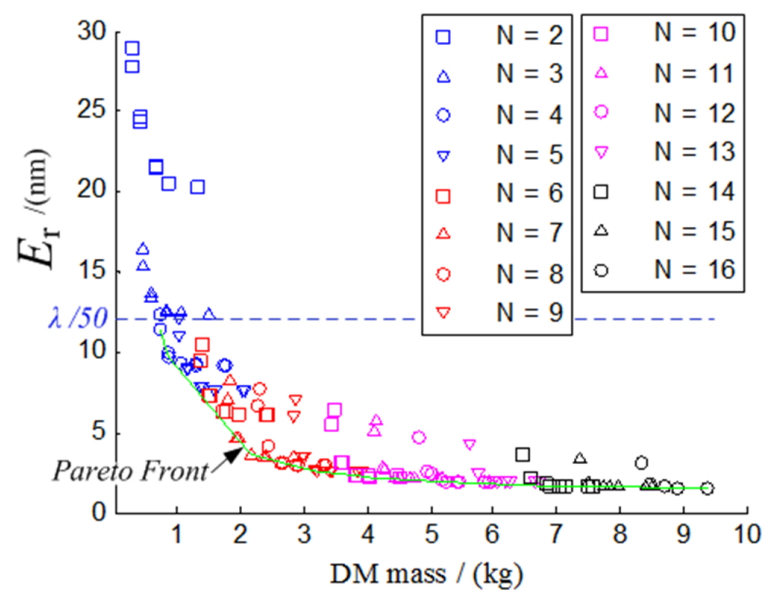

FIG. 11. Trade space for a triangularly configured DM.

TABLE 5. Pareto optimal designs for a triangularly configured $\mathrm{DM}$

\begin{tabular}{c|c|c|c|c}
\hline \hline$N$ & $T[\mathrm{~mm}]$ & $\phi[\mathrm{mm}]$ & Mass $[\mathrm{kg}]$ & $E_{\mathrm{r}}[\mathrm{nm}]$ \\
\hline 4 & 0.4 & 4 & 0.71 & 11.39 \\
\hline 4 & 0.4 & 8 & 0.84 & 9.77 \\
\hline 5 & 1 & 4 & 1.14 & 8.92 \\
\hline 5 & 2 & 6 & 1.38 & 7.80 \\
\hline 6 & 1 & 4 & 1.51 & 7.30 \\
\hline 6 & 2 & 4 & 1.73 & 6.30 \\
\hline 7 & 1 & 4 & 1.93 & 4.65 \\
\hline 7 & 2 & 4 & 2.16 & 3.63 \\
\hline 8 & 2 & 6 & 2.67 & 3.06 \\
\hline 9 & 2 & 6 & 3.22 & 2.61 \\
\hline 10 & 2 & 6 & 3.83 & 2.30 \\
\hline 11 & 2 & 6 & 4.51 & 2.15 \\
\hline 12 & 3 & 4 & 5.41 & 1.95 \\
\hline 13 & 3 & 6 & 6.27 & 1.88 \\
\hline 14 & 3 & 6 & 7.13 & 1.69 \\
\hline 15 & 5 & 4 & 8.40 & 1.62 \\
\hline 16 & 3 & 4 & 8.93 & 1.57 \\
\hline
\end{tabular}

The relationship between $N$ and the number of actuators is shown in Table 6. The parameter $N$ is dominant in the correction capability of the DM. Increasing $N$ from 2 to 16 (and the corresponding number of actuators from 19 to 817 ) decreases $E_{r}$ from $27.8 \mathrm{~nm}$ to $1.7 \mathrm{~nm}$-a $94 \%$ reduction.

Note that the parameter $T$ also has a significant effect on the two metrics of the DM. Generally, increasing the thickness of the DM causes $E_{r}$ to decrease. This can be explained by the print-through effect: Dimpling of the mirror is reduced as the local stiffness around the actuator increases due to the increasing thickness of the DM. Similarly, better correction capability may be obtained through thicker
TABLE 6. Relation between $N$ and the number of actuators

\begin{tabular}{|c|c|c|}
\hline$N$ & $\begin{array}{l}\text { No. of actuators } \\
\text { (triangularly } \\
\text { configured DM) }\end{array}$ & $\begin{array}{c}\text { No. of actuators } \\
\text { (squarely } \\
\text { configured DM) }\end{array}$ \\
\hline 1 & - & 9 \\
\hline 2 & 19 & 25 \\
\hline 3 & 37 & 49 \\
\hline 4 & 61 & 81 \\
\hline 5 & 91 & 121 \\
\hline 6 & 127 & 169 \\
\hline 7 & 169 & 225 \\
\hline 8 & 217 & 289 \\
\hline 9 & 271 & 361 \\
\hline 10 & 331 & 441 \\
\hline 11 & 397 & 529 \\
\hline 12 & 469 & 625 \\
\hline 13 & 547 & 729 \\
\hline 14 & 631 & 841 \\
\hline 15 & 721 & 961 \\
\hline 16 & 817 & 1089 \\
\hline
\end{tabular}

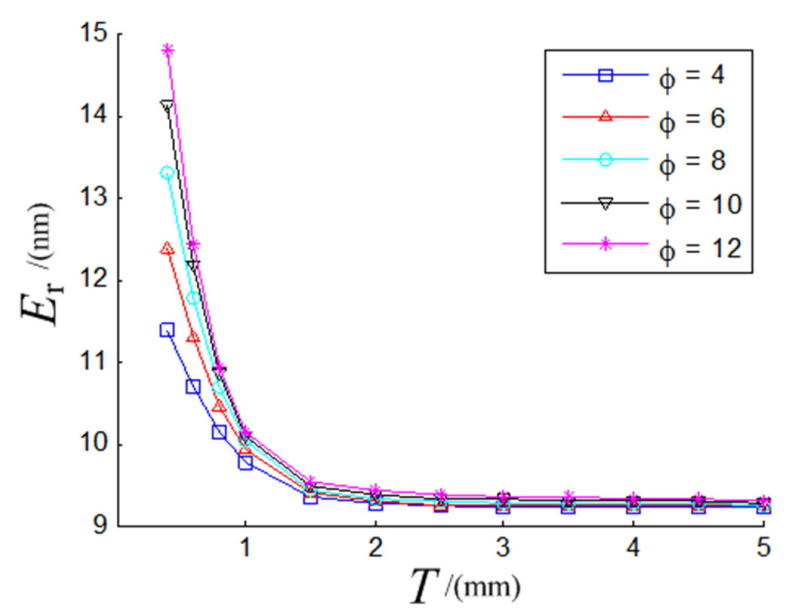

FIG. 12. Impact of the correction capability for various $\phi$ over $T$ with a triangularly configured DM.

mirror design. Hence, an alternative design may be seen for the same metrics of mass and $E_{r}$.

To examine the effects of parameters $T$ and $\phi$ closely, by assuming $N=4$ and varying $T$ from $0.4 \mathrm{~mm}$ to $5 \mathrm{~mm}$ and $\phi$ from $4 \mathrm{~mm}$ to $12 \mathrm{~mm}$, the associated DM correction capability $E_{r}$ is analyzed. The results are shown in Fig. 12 for the triangularly configured DM. Note that increasing $T$ from $0.4 \mathrm{~mm}$ to $5 \mathrm{~mm}$ decreases $E_{r}$ from $14.8 \mathrm{~nm}$ to 9.3 $\mathrm{nm}-\mathrm{a} 37 \%$ reduction, while increasing $\phi$ from $4 \mathrm{~mm}$ to 12 $\mathrm{mm}$ increases $E_{r}$ from $11.4 \mathrm{~nm}$ to $14.8 \mathrm{~nm}-\mathrm{a} 30 \%$ rise at most. By increasing $T$, the influence of $\phi$ on correction 


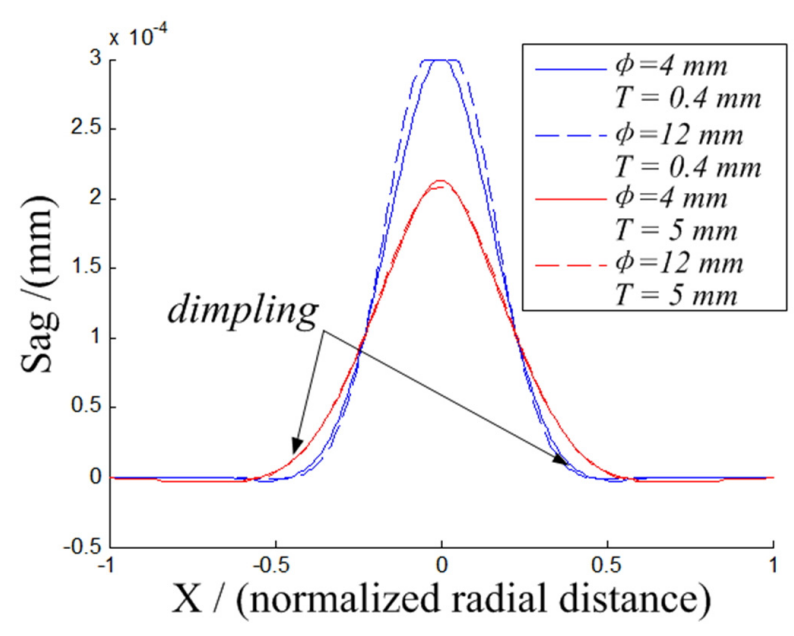

FIG. 13. Influence function comparison with $T$ and $\phi$.

capability is attenuated to zero, and it matches the actuator print-through effect well. As shown in Fig. 13, for a DM with a thin mirror $(T=0.4 \mathrm{~mm})$ the local area stiffness is quite sensitive to the additional stiffness due to the pad; hence the influence function (i.e., sag of the mirror) is greatly flattened for a DM with a largePad $(\phi=12 \mathrm{~mm})$, which attenuates the DM's correction capability. The pad is used for alleviation of sudden changes and stress concentrations in the actuator-mirror connection by strengthening the local structure. Although a smaller pad design delivers better DM performance and lighter weight, the pad should be large enough for manufacturing concerns. Meanwhile, the dimpling induced by activation is much clearer for a DM with a thin mirror compared to a DM with a thick mirror $(T=5$ $\mathrm{mm}$ ), which attenuates the DM's correction capability as well, so that DMs with thick mirrors tend to work better for figure error correction.

\subsection{Variation of Actuator Configuration}

It has been believed that the triangularly configured DM is space-efficient in application and possesses higher performance than DMs with other kinds of actuator configurations. It seems that the triangularly configured DM is more suitable for the specific application discussed in this paper, since the WFE requiring correction comprises many trefoil WFEs. As another candidate, a squarely configured $\mathrm{DM}$ is analyzed. The trade space for the squarely configured DM is presented in Fig. 14. Similar trends can be found: The correction capability metric $E_{r}$ decreases as the number of actuators $(N)$ increases. The best designs will be the ones under the trade-off between $E_{r}$ and the mass. The Pareto Front is plotted as a green line, and the Pareto Optimal Designs are listed in Table 7.

To compare the two different actuator configurations, only the two Pareto Fronts and Pareto Optimal Designs for each case are illustrated in Fig. 15(a). Accounting for the noncircular shapes of these two kinds of DM, a DM trimmed into a circle will lose some mass. Although the manner is

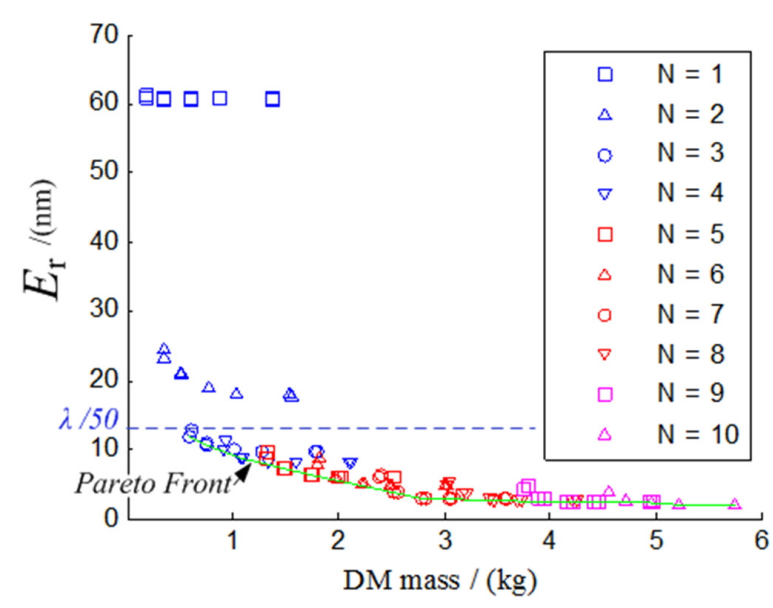

FIG. 14. Trade space for a squarely configured DM.

TABLE 7. Pareto optimal designs for a squarely configured DM

\begin{tabular}{c|c|c|c|c}
\hline \hline$N$ & $T[\mathrm{~mm}]$ & $\phi[\mathrm{mm}]$ & Mass $[\mathrm{kg}]$ & $E_{\mathrm{r}}[\mathrm{nm}]$ \\
\hline 3 & 0.4 & 4 & 0.60 & 11.86 \\
\hline 3 & 1 & 4 & 0.75 & 10.65 \\
\hline 4 & 0.4 & 8 & 1.08 & 8.68 \\
\hline 4 & 0.4 & 6 & 1.34 & 8.08 \\
\hline 5 & 1 & 4 & 1.48 & 7.23 \\
\hline 5 & 2 & 4 & 1.74 & 6.24 \\
\hline 6 & 1 & 4 & 1.97 & 6.00 \\
\hline 6 & 2 & 6 & 2.25 & 5.01 \\
\hline 7 & 1 & 4 & 2.53 & 3.94 \\
\hline 7 & 2 & 4 & 2.79 & 3.00 \\
\hline 8 & 2 & 6 & 3.47 & 2.77 \\
\hline 9 & 2 & 6 & 4.21 & 2.37 \\
\hline 10 & 2 & 4 & 4.97 & 2.18 \\
\hline
\end{tabular}

rough, it can deliver some insights for application. The result for a trimmed DM is shown in Fig. 15(b). The difference between the two kinds of DM is relatively small, a squarely configured DM being slightly better than the triangularly configured one in Fig. 15(b). It can be explained by realizing that squarely configured DMs have more versatile changes in azimuthal order. However, since the difference is relatively small, it can be concluded that the type of configuration of DM is not a sensitive parameter in design; application and fabrication concerns take priority.

\section{CONCLUSION}

A parametric modeling method is introduced in this paper, making practical and flexible the design and optimization of DMs used in large-aperture optical systems. The parameter 


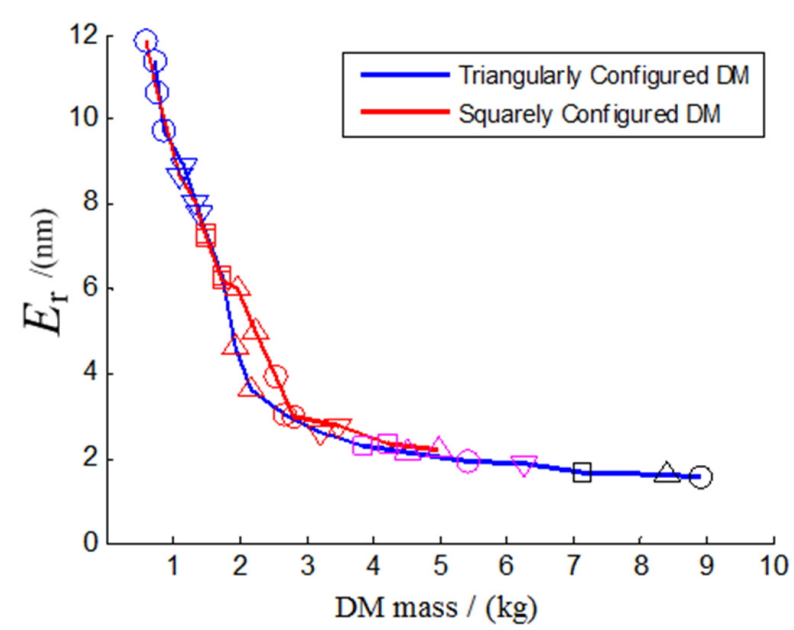

(a)

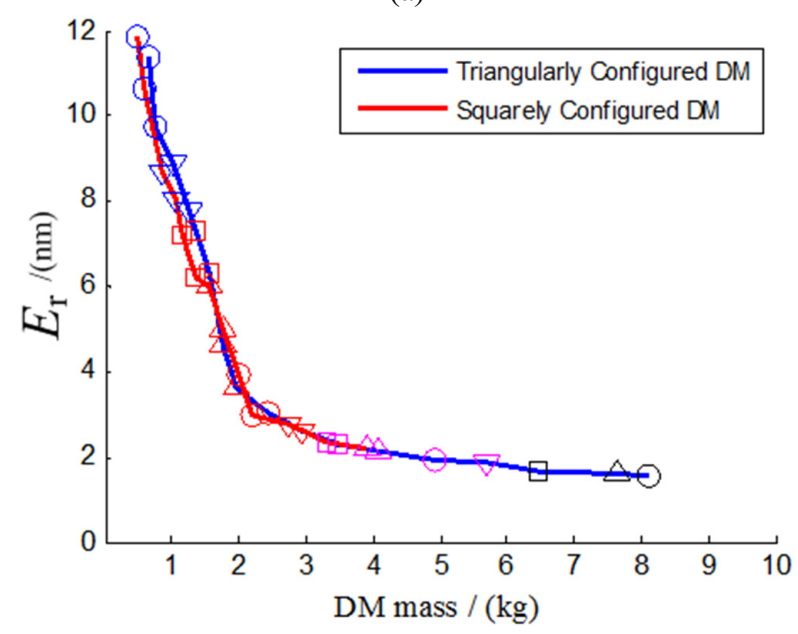

(b)

FIG. 15. Pareto Front comparison for triangularly and squarely configured DMs: (a) without trim, (b) with trim.

trade-off, which used to be impractical due to the time cost of manual-only meshing in large, complex models, is realized by a combination of manual meshing and autogeneration of FEM. Furthermore, the method is capable of dealing with other parametric modeling applications.

The metrics for a DM discussed in this paper are the correction capability and the mass of DM. The correction capability of a DM is represented by the residual WFE for specific figure correction, and the mass represents the launch cost concern. The optimal design has the best correction capability and the smallest mass. To find the interaction between variations of design parameters and the metrics of a DM, a full trade space is analyzed for triangularly and squarely configured DMs, providing design guidelines and insight. The results show that the correction capability of a DM increases as the mirror thickness $T$ and number of actuators $N$ increase, or the size of the pad $\phi$ decreases. However, the size of the pad has a lower limit for manufacturing concerns, and increasing either $T$ or $N$ adds mass to the DM. Hence, the best design will be a trade-off between the correction capability and the mass, and will vary depending on the specific application. It is demonstrated that the difference of correction capability for various actuator configurations is relatively small, and the configuration type of a DM is not a sensitive parameter in its design.

\section{ACKNOWLEDGMENT}

This work is funded by the National High Technology Research and Development Program of China (863 Program) (No. 863-2-5-1-13B).

\section{REFERENCES}

1. L. Feinberg, L. Cohen, B. Dean, W. Hayden, J. Howard, and R. Keski-Kuha, "Space telescope design considerations," Opt. Eng. 51, 011006 (2012).

2. S. E. Forman, "Advanced Land Imager: mechanical design, integration, and testing," Lincoln Laboratory Journal 15, 181-196 (2005).

3. H. P. Stahl, M. Postman, and W. S. Smith, "Engineering specification for large-aperture UVO space telescopes derived from science requirements," Proc. SPIE 8860, 886006 (2013).

4. M. D. Lallo, "Experience with the Hubble Space Telescope: 20 years of an archetype," Opt. Eng. 51, 011011 (2012).

5. P. Schipani, S. D'Orsi, L. Ferragina, D. Fierro, L. Marty, C. Molfese, and F. Perrotta, "Active optics primary mirror support system for the $2.6 \mathrm{~m}$ VST telescope," Appl. Opt. 49, 1234-1241 (2010).

6. L. D. Feinberg, M. Clampin, R. Keski-Kuha, C. Atkinson, S. Texter, M. Bergeland, and B. B. Gallagher, "James Webb Space Telescope optical telescope element mirror development history and results," Proc. SPIE 8442, 84422B (2012).

7. J. J. M. Oschmann, G. Hickey, T. Barbee, M. Ealey, D. Redding, M. C. Clampin, and H. A. MacEwen, "Actuated hybrid mirrors for space telescopes," Proc. SPIE 7731, 773120 (2010).

8. M. Postman, W. B. Sparks, F. Liu, K. Ess, J. Green, K. G. Carpenter, H. Thronson, and R. Goullioud, "Using the ISS as a testbed to prepare for the next generation of space-based telescopes," Proc. SPIE 8442, 84421 T (2012).

9. M. Laslandes, E. Hugot, M. Ferrari, C. Hourtoule, C. Singer, C. Devilliers, C. Lopez, and F. Chazallet, "Mirror actively deformed and regulated for applications in space: design and performance," Opt. Eng. 52, 091803 (2013).

10. Z. Challita, T. Agócs, E. Hugot, A. Jaskó, G. Kroes, W. Taylor, C. Miller, H. Schnetler, L. Venema, L. Mosoni, D. Le Mignant, M. Ferrari, J.-G. Cuby, "Design and development of a freeform active mirror for an astronomy application," Opt. Eng. 53, 031311 (2014).

11. S. Ravensbergen, R. Hamelinck, P. Rosielle, and M. Steinbuch, "Deformable mirrors: design fundamentals for force actuation of continuous facesheets," Proc. SPIE 7466, 74660G (2009).

12. M. W. Smith and D. W. Miller, "Minimizing actuator-induced errors in active space telescope mirrors," Proc. SPIE 7731, 773122 (2010).

13. L. E. Cohan, "Integrated modeling for design of lightweight, 
active mirrors," Opt. Eng. 50, 063003 (2011).

14. W. R. Arnold, R. M. Bevan, and H. P. Stahl, "Integration of mirror design with suspension system using NASA's new mirror modeling software," Proc. SPIE 8836, 88360J (2013).

15. S. A. Mitchell, M. A. Mohammed, A. H. Mahmoud, and M. S. Ebeida, "Delaunay quadrangulation by two-coloring vertices," Procedia Engineering 82, 364-376 (2014).

16. L. E. Cohan. "Integrated modeling and design of lightweight, active mirrors for launch survival and on-orbit performance," Ph.D. Thesis, Massachusetts Institute of Technology (2010).

17. S. A. Uebelhart, "Non-deterministic design and analysis of parameterized optical structures during conceptual design," Ph.D. Thesis, Massachusetts Institute of Technology (2006). 